\title{
Mixed Infection due to Plasmodium malariae and Plasmodium vivax: A First Case Report from Rural North India
}

\author{
Pratibha Mane, Jyoti Sangwan*
}

Pratibha Mane, Jyoti Sangwan*

Department of Microbiology,

SHKM,GMC, Mewat, Haryana, INDIA.

\section{Correspondence}

Dr. Jyoti Sangwan

Associate Professor, Department of Microbiology, SHKM,GMC, Mewat, Haryana, INDIA.

Mobile no: +91 8199972272

Email: jyolathwal@yahoo.co.in

History

- Submission Date: 28-07-2020

- Revised Date: 28-08-2020

- Accepted Date: 24-09-2020

DOI : 10.5530/ijmedph.2020.4.48

Article Available online

http://www.ijmedph.org/v10/i4

\section{Copyright}

(C) 2020 Phcog.Net. This is an openaccess article distributed under the terms of the Creative Commons Attribution 4.0 International license.

\begin{abstract}
Mixed malaria infections often go unrecognized and are therefore under reported. We hereby report the first described case of mixed infection due to $P$. malariae and $P$. vivax from most backward region of country emphasizing possibility of change in epidemiological pattern of malaria in the region. Also present case report highlight the increasing importance of Polymerase Chain Reaction in diagnosing malaria especially mixed malaria.
\end{abstract}

Key words: Malaria, Plasmodium malariae, Plasmodium falciparum, Polymerase Chain Reaction in diagnosing.

\section{INTRODUCTION}

Malaria is one of the most important parasitic infection throughout the world and it results in high mortality rates. The causative agent Plasmodium spp. are transmitted by Anopheles mosquitoes. ${ }^{1}$ Human malaria is a protozoan infection caused by five Plasmodium species which includes $P$. falciparum, $P$. vivax, P. ovale, P. malariae and P. knowlesi. All species cause the disease which is different in transmission, nature of immune response, clinical manifestation and response to drugs. ${ }^{2-4}$ National vector borne disease control program estimate about $2-3$ million cases of malaria occur annually in India. ${ }^{5}$

The most prevalent species causing disease in India is $P$. vivax accounting for about $65 \%$ of cases and next common species is $P$. falciparum which is responsible for about $35 \%$ of cases. The remaining species are infrequently seen in India with few cases sprouting from different states of India such as Orissa, West Bengal, Madhya Pradesh, Karnataka, Tamil Nadu, Kerala, Arunachal Pradesh and Assam., ${ }^{2,-10}$ P. ovale is the rarest infection with only four cases reported in India, one each from Kolkata, Orissa, Delhi and Assam. ${ }^{11}$ To best of our knowledge, we are reporting probably the first case of mixed infection by $P$. malaria and $P$. vivax from Haryana.

\section{CASE REPORT}

A 50-yr old man from Nuh, Haryana walked in outpatient department with complaints of fever, headache and abdominal pain. There was no history of travel to other state of India. On examination, pallor was present, abdomen was soft with diffuse tenderness. Other systems were within normal limits He was evaluated for fever. Laboratory tests showed Hemoglobin - 9 gm/dl. Other parameters were within normal limits. Thick and thin blood smears were prepared. Microscopic examination of blood smears showed band form, ring forms and gametocytes of malarial parasite. (Figure 1). On microscopy a diagnosis of $P$. malariae was made due to the size of infected RBCs being same as that of uninfected ones. Also presence of malarial gametocytes were observed. On the basis of these findings, a diagnosis of Malaria due to P. malariae was made. Since the presence of $P$. malariae in this region was not reported before, it was decided to send the sample to National Institute of Malaria Research, New Delhi for confirmation. Polymerase Chain Reaction (PCR) was used for confirmation and presence of $P$. malariae along with $P$. vivax was confirmed in the blood sample. The results underline the possibility of a co-infection by the two species. The gametocytes of two species resemble each other so probably missed on microscopy. The patient was treated under supervision for uncomplicated malaria with oral artesunate plus sulphadoxinepyrimethamine for three days. Fever subsided on the $3^{\text {rd }}$ day after the initial examination, the daily dose continued for seven days and the patient remained afebrile until the day of discharge.

\section{DISCUSSION}

This is probably first case report of $P$. malariae from Haryana which was also confirmed by PCR. The estimated prevalence of $P$. malariae - P. vivax mixed infections is documented to be less than $3 \% .{ }^{12}$

During microscopic examination of thick blood film, the morphology of $P$. malariae can be confused with $P$. vivax. The ring forms of the two species are so similar that Garnham described the ring forms of $P$. malariae in blood films as "rather like those of $P$. vivax although less amoeboid and with a more dense 


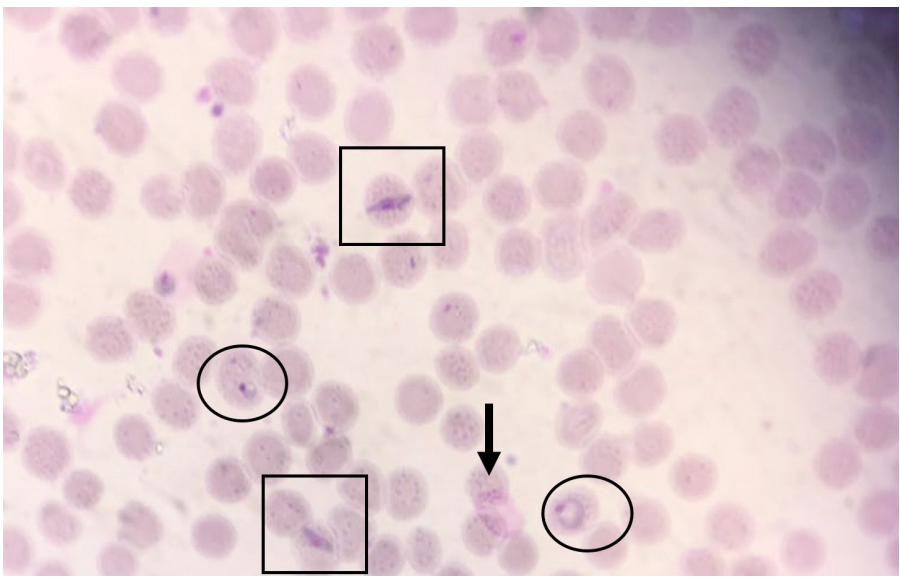

Figure 1: Band forms and Ring forms (Size of infected RBCs is same that of uninfected) and Gametocytes of P. malariae. Magnification 10x100

ring of cytoplasm". However, size of infected RBCs can be crucial while arriving at a diagnosis. Under NVBDCP, Jaswant Singh and Bhattacharyya (JSB) stain is used for routine malaria microscopy examination of thick and thin smear. The morphological changes induced by hemolysis can hamper the identification of infecting species or inadequate knowledge and experience of the reporting personnel to identify malaria parasites other than $P$. falciparum and $P$. vivax, diagnosis of atypical parasites are missed. Moreover, $P$. malariae and $P$. ovale species are difficult to identify if the typical morphology of the infected host cell is damaged. On microscopy P. malariae in India remains underestimated. PCR based identification method is a confirmatory diagnostic tool. But the facility remain largely lacking in rural and remote areas like ours. ${ }^{2}$

P. malariae is known for its recurrent infection potential and propensity to develop nephrotic syndrome in patients. $P$. malariae can remain for decades within a human host and in a state potentially infectious to mosquitoes thus, facilitating the transmission of this species over a long period of time. ${ }^{2,13}$

P. malariae rapidly responds to routinely used antimalarial drugs and is not known to cause severe clinical disease. Our patient also responded well to the treatment. To control malarial infection, successful treatment is required, as incomplete treatment can lead to the development of resistance and recrudescence. Choloroquine drug resistance is common in Plasmodium spp. Now artemisinin-based combination therapies are used for $P$. falciparum and mixed malaria infections. This regimen consists of doxycycline $100 \mathrm{mg} / \mathrm{BD}$ and artesunate $2.4 \mathrm{mg} / \mathrm{kg}$, intravenously at 0 , 12 and $24 \mathrm{hr}$, then daily for seven days and the addition of primaquine $30 \mathrm{mg} /$ day on the third day, for a period of three days, to eradicate the gametocytes. Mixed malaria species infection is common, but to control the disease early diagnosis and appropriate treatment is required. ${ }^{1}$

\section{CONCLUSION}

Although $P$. malariae do not cause sever disease but its presence in this part of country indicates change in epidemiological pattern of malaria infection. This region of Haryana is endemic for malarial infection and complicated cases of cerebral malaria are not uncommon. Even though the morphology was quite characteristic of $P$. malariae, making diagnosis possible, on PCR simultaneous presence of $P$. vivax was also confirmed. Geographical region should not be deterrent for doing PCR; as dynamics of human movement in an area can spread the infection.

\section{ACKNOWLEDGEMENT}

A heartfelt gratitude to Dr. Anoop Anvikar, Scientist and technical staff, National Institute of Malaria Research (ICMR), Dwarka, New Delhi for confirmation of the species.

\section{CONFLICT OF INTEREST}

The authors declare that there is no conflict of interest.

\section{ABBREVIATIONS}

PCR: Polymerase chain Reaction; JSB: Jaswant Singh and Bhattacharyya; ICMR: Indian Council of Medical Research.

\section{REFERENCES}

1. Mohammad Y, Davood Y, Amirhossein MM, Mahmood N. Mixed Infection of Plasmodium malariae and Plasmodium falciparum: A Case Report. Arch Clin Infect Dis. 2014;9(1):e19297.

2. Mohapatra PK, Prakash A, Bhattacharyya DR, Goswami BK, Ahmed A, Sarmah $B$, et al. Detection and molecular confirmation of a focus of $P$. malariae in Arunachal Pradesh. Indian J Med Res. 2008;128(1):52-6.

3. Singh B, Kim SL, Matusop A, Radhakrishnan A, Shamsul SS, Cox-Singh J, et al. A large focus of naturally acquired Plasmodium knowlesi infections in human beings. Lancet. 2004;363(9414):1017-24.

4. Liu Q, Zhu S, Mizuno S, Kimura M, Liu P, Isomura S, et al. Sequence variation in the small-subunit rRNA gene of Plasmodium malariae and prevalence of isolates with the variant sequence in Sichuan, China. J Clin Microbiol. 1998;36(11):3378-81.

5. Sharma VP. Ecosystem approach to malaria control. Proc Natl Acad Sci India. 1993;63(B):47-55

6. Das S, Malakar P, Saha GK, Dasgupta B, Hati AK. A case of Plasmodium malariae infection in the Dooars region of West Bengal, India. Indian J Malariol. 1996;33(3):159-60.

7. Panda R, Verma KV, Rahman SJ. Present status of Plasmodium malariae infection in Bastar district (M.P). J Commun Dis. 1990;22(3):185-90.

8. Roy RG, Shanmugham CAK, Chakrapani KP, Madesayya NM, Ghosh RB. Distribution of human plasmodia in Karnataka and Tamil Nadu States in the reappearing phase of malaria in 1970 and 1975. Indian J Med Res. 1979;69:539

9. Roy RG, Chakrapani KP, Zacharias J. Plasmodium malariae in Kerala and Tami Nadu States of India (1969-75). Trans R Soc Trop Med Hyg. 1977;71(5):447.

10. Dev V. Plasmodium malariae - a case of quartan malaria in Assam. J Commun Dis. 2000;32(2):149-51.

11. Prakash A, Mohapatra PK, Bhattacharya DR, Goswami BK, Mahanta J. Plasmodium ovale: First case report from Assam. Curr Sci. 2003;84(9):1187-8.

12. Imirzalioglu C, Soydan N, Schaller M, Bretzel RG, Chakraborty T, Domann E. Diagnosis of mixed Plasmodium malariae and P. vivax infection in a development aid volunteer by examination of bone-marrow specimens by real-time PCR. J Clin Microbiol. 2006;44(6):2307-10.

13. Savargaonkar D, Shah N, Das MK, Srivastava B, Valecha N. Plasmodium malariae infection: A case of missed diagnosis. J Vector Borne Dis. 2014;51(2):149-51. 